\title{
SOME PRACTICAL POINTS IN THE MANAGEMENT OF AN ISOLATION HOSPITAL.
}

\author{
By A. KNYVETT GORDON, B.A., M.B, B.C. Cantab. \\ Medical Superintendent of,Monsall Hospital ; Lecturer on Infectious Diseases \\ in the University of Manchester.
}

I PROPOSE to-night to put before you, more as suggestion for discussion than as a dissertation, a few points which $I$ have come to consider essential in the internal administration of a large fever hospital. As there is not time to-night to state the reasons which have led to these conclusions, the latter must of necessity appear somewhat dogmatic. For this semblance I must crave your indulgence.

The first point that I shall refer to is the importance of asepsis, or surgical cleanliness, and the methods by which one seeks to attain it.

Now, in an isolation hospital there are patients suffering from different infections: To Monsall, for instance, besides searlet fever, enteric fever, and diphtheria, cases of puerperal fever, erysipelas, and tuberculosis are admitted, and it is obvious that these diseases must be separated from one another, and care must be taken to prevent cross infection; and it would not be too much to say that the extent to which superadded disease occurs in any hospital is in a large degree a measure of its efficiency.

But it is no less true that one patient is often infectious to another suffering ostensibly from the same disease. If, for instance, we examine the throats of half a dozen cases of scarlet fever in the same ward, it is seldom that we find the same assortment of organisms in any two of them. If one of these patients happen to have diphtheria bacilli in the fauces, and another patient contracts true diphtheria, we call it post-scarlatinal diphtheria, and we know it to be a superadded infection and a preventible one; but if a child comes in with a mild faucial affection, from which staphylococci only are grown on culture, and on the admission to the ward of another case with a more severe attack his throat becomes worse, and is then found to contain one or more varieties of streptococci also, we are wont to call this the natural course of events, or at most a "complication" of the disease, and we do not as a rule consider it in the light of a preventible occurrence. Yet I must confess that I see no difference in the etiology of the two infections.

It is necessary, then, that everybody in the hospital should understand that any one case may be infectious to any other, whether they are in the same ward or not; from this it arises that when any such 
infection occurs, rigid enquiry should be made to discover how it has arisen. Most of the complications of scarlet fever we know to be due to superadded infection, while we suspect this may be so in some others. How does infection occur? I think we may take it that the main factor with any disease is transmission of infective matter, either directly from an unrecognized case, or indirectly from infected dust, by hands, clothing, and instruments. To a much lesser extent there is transference by aërial convection within the ward; but I must confess that I am myself very sceptical as to the occurrence of this, at all events to the extent to which it is popularly supposed to happen. It is obviously such a very convenient harbour of refuge in which dirty hands may shelter themselves from the storm of an official enquiry.

I am doubtful, too, as to whether infection lingers much on the walls, tables, etc., in a ward, when they are clean to the naked eye. One would imagine from the writings of some, mostly architects I think, that the be-all and end-all of an isolation hospital was aseptic wards and furniture. Far be it from me to minimize the importance of these; but authorities have frequently spent large sums on the construction of a hospital, and have economized in the silliest way in intelligence or number of the staff. To my mind the staff are more potent factors for good or evil in dealing with infection, than any amount of glazed tiles and glass tables. Curiously enough, architects have frequently designed most modern hospitals with walls, ceilings, and tables all one could wish, and have constructed the floor, surely the most important part of all, if only because the infection ultimately gets there, of more or less porous wood, entailing infinite labour in wet sweeping to keep it free from dust, and in subsequent polishing to improve its appearance. I should personally prefer a floor made of terrazo, concrete, or some other impervious substance that could be flushed with water from a hose, and then dried with a rubber squeegee, so that no sweeping (either wet or dry) would ever be required.

I would then premise that the most important point is to eliminate what I- will call staff infection; and by that I mean transference of infective matter by hands, instruments, and clothing of doctors or nurses, chiefly the latter, because their contact with the patient is more intimate and more prolonged. Let me then mention in some detail the precautions that I have come to consider advisable to attain that end. It is first necessary to try and diminish the extent to which infective matter is manufactured, and there can be no doubt that the most infectious things in a ward are the throats of the patients with scarlet fever and diphtheria, and the excreta of those with enteric fever.

I do not propose to-night to deal with the methods of treating 
excreta; that ground is well-trodden already, but I may state in passing that I regard izal as by far the best disinfectant for the purpose.

But I think it is a point of the first importance to render the throats of scarlet fever patients as innocuous as possible to their surroundings. With diphtheria, it is seldom safe, on account of the circulatory depression which the disease produces, to attempt any adequate disinfection of the throat. The method that I usually employ in the anse of scarlet fever is to swab the fauces in all cases where there is much ulceration, especially if the odour of the breath is offensive, with undiluted izal. I have found that this drug has very little action on healthy mucous membrane, but that it attacks energetically dead and decomposing tissue. The mouth is held open with a gag, the fauces well exposed and dried, and then pure izal is deliberately applied to the ulcerated surfaces, any superfluous fluid being removed by a large wet swab. While I commenced by adopting this method solely with a view of curing the particular patient, I found subsequently that the treatment of the severe cases in this way was an advantage to the rest of the patients in the ward. Clinically the effect of this drug is often very well marked, and its selective action on necrotic tissue is of great value. As far as my experience goes, and it is now fairly extensive, I have not found this selective action to occur with other disinfectants.

The next point is to make sure that in the proc ss of treatment, infective matter is not conveyed from one patient to another. Here there are two essentials : firstly, it is not safe to use any kind of syringe for the treatment of the throat, especially those made of india-rubber; secondly, the hands of the nurse must be sterile and must remain so.

At Monsall, a travelling dressing waggon is employed, which carries sufficient material for the cleansing of all the throats in the ward. The bone nozzles, one for each patient, are boiled and placed in a solution of boracic acid, and the swabs made of cotton wool are similarly treated. The nurse sterilizes her hands by scrubbing them with soft soap and water, followed by the use of turpentine and methylated spirit. She then puts on a pair of rubber gloves, which have been recently boiled-in practice, nozzles, swabs and gloves are all boiled together-and proceeds to the treatment of the first patient. When he has been attended to, the gloved hands are cleansed by holding them under the tap for a few minutes, followed by immersion in a solution of izal of the strength of 1 in 200 of water. By this means it is practically impossible for infection to be conveyed by the instruments or hands employed in the process of the throat treatment. The hands are, moreover, not rendered hard and chapped by frequent 
immersion in antiseptic solutions. I may say in passing that rubber gloves are worn by the nurses whenever they touch an enteric patient or any contaminated linen. Another essential point is that the nurses should wear their sleeves rolled up above the elbow whenever they are doing anything for a patient; the cuffs of a dress can both hold infective matter and transfer it from one patient to another. Similarly, the medical men while in the wards wear a washable suit of white drill; not, by the bye, a loose overall. Another important point is the treatment of discharging ears; when otorrhoea exists, the risk of a patient infecting his surroundings is enormously increased, as the pillow and bed-clothes become soaked with the discharge. Consequently all discharging ears are kept plugged with a loose wad of wool soaked in weak izal or other reliable antiseptic, and then covered with a pad of wool and a bandage. Providing that the plug is not too tight, and is frequently changed, the healing of the otitis is not delayed; irritation of the meatus is prevented by changing the antiseptic occasionally, and by keeping the parts well smeared with lanoline, or other ointment. If this method is adopted, cases which have diphtheria bacilli in the aural discharge can be safely treated in the ordinary ward. Where, however, they are present in the nasal discharge (without symptoms of true diphtheria) the patient is isolated.

I have said that superadded infection proceeds originally from an unrecognized case. It may be as well, then, briefly to mention the precautions that are taken at Monsall to ensure as far as possible a correct diagnosis.

The first point is that no case should be admitted to any ward unless one is certain from one's own actual observation that the patient is suffering from the disease certified. One cannot diagnose disease from the history, even when one sees oneself undoubted cases of the disease certified in other members of the family. In the case of scarlet fever especially, one has to remember that it is a mixed infection in practice, whatever it may be in theory, and that the assortment of organisms in any two cases is seldom the same. I believe that from a case of scarlet fever, other members of the family may catch either scarlet fever (of course the more common occurrence), or what we know as a septic throat, or some other streptococcal manifestation.

So cases are examined thoroughly in the ambulance before they are sent to a ward at all. It seems almost silly to mention this fact; but there are hospitals, I am sorry to say, where cases are sent without examination to the wards for the disease certified, and are examined there some hours later. This is, I submit, an absolutely unjustifiable practice. A minor point is that $I$ do not personally like receiving 
rooms. My experience with them in the past has been that you may disinfect the room to any extent after the examination of a doubtful case ; but you cannot get the nurse to apply an allied process to herself, especially when, as is often the case, she has been selected for that work because her days of usefulness in the ward are past. Here again, hands and clothing are the important factors.

If a patient comes in, then, who has not obvious positive signs of the certified disease, he is sent to a small isolation ward, and the medical man who certified him is written to in order that as full a history as possible may be obtained, and he is not sent to one of the general wards until some positive sign of the particular disease develops, and he is kept in until it is certain that he could not have had the disease in question; in practice a period of about three weeks usually suffices for this purpose.

A special point in connection with diagnosis is the elimination of post-scarlatinal diphtheria as far as possible, by extensive bacteriological examination of anything at all suspicious, in the hospital laboratory. Here, all patients that have bacilli morphologically indistinguishable from diphtheria bacilli are considered to be sources of infection, though they are not necessarily labelled cases of diphtheria clinically. In all probability the great majority of these bacilli are not diphtheria bacilli at all, but we cannot affirm this in any given case; it does not help us even to have the virulence determined, as this is so changeable a factor in the case of true diphtheria bacilli. Any bacillus may be saprophytic merely in one patient, and parasitic in another. As an administrative procedure, I a.m convinced that the isolation of these patients is a matter of vital importance, though I strongly object to their being labelled for statistical purposes as cases of true diphtheria.

It is, of course, essential that the walls, and floors, and furniture of the ward shall be kept clean by damp rubbing, not dusting. When a superadded infection has occurred to any patient in the ward, all the cups, feeding bottles, spoons, forks, and plates are boiled, and everything that cannot be boiled eonveniently is scrubbed with strong izal solution. Incidentally, I cannot see why we should continue to employ carbolic acid and perchloride of mercury for gross disinfection when we have in izal such a much better germicide, and personally I only use them occasionally in the treatment of wounds, and in some operations.

It will be obvious from the foregoing remarks that bacteriological work must enter very largely into the routine of a modern fever hospital, and I need hardly point out that it is essential that the laboratory should be in the hospital itself, so that any investigation 
may be made at once. It is not possible to detect superadded infection in time for the result to be of any practical use, if the specimens are sent away to a laboratory outside.

At Monsall we have derived great advantage from the examination of fresh specimens, though the results are always checked by subsequent cultures. On the admission of a patient alleged to be suffering simply from scarlet fever, for instance, but whose throat looks suspicious, a smear specimen is made and examined before the patient is removed from the ambulance; it has repeatedly happened that we have been able to detect co-existent diphtheria in this manner, and the introduction of the patient to an uninfected ward has been prevented. Similarly, when in convalescence from scarlet fever a patient develops a sore throat, the immediate results obtained from the examination of a fresh specimen are often invaluable. Of course, in this connection, a negative result cannot be relied upon as showing the absence of any particular micro-organism.

At Monsall every nasal or aural discharge in a searlet fever ward is examined bacteriologically when it arises, and subsequently at frequent intervals. Cultures are also made from the nose and ears when the patient is ready to go, even though the discharge may have stopped; and the examination of these cultures forms part of the routine investigation before the patient is considered fit to be sent home. I do not say free from infection, for I do not know when any patient who has had scarlet fever is free from infection. Another essential, in my opinion, in a fever hospital is an aseptic operating theatre. To operate in cases that are often septic to an extreme degree in an ordinary ward, is to court the spread of infection by the hands and clothing of those assisting. If the walls, floor, and fittings of the theatre are impervious, it is perfectly safe to take any patient into it without any risk of infecting the next case that goes there, and $I$ have never found such infection occur. Moreover, there are many cases, especially of enteric fever and puerperal septicæmia, to say nothing of the surgical affections that are sometimes sent in erroneously as enteric fever, where a major operation is indicated, and this cannot be done with safety or the necessary rapidity, on a bed in the ward.

I have said that it is on the nursing staff that the extent to which surgical cleanliness is possible must mainly depend. To get the best results it is essential to engage young women and train them. That, again, is only possible when the hospital can give a certificate of training that is recognized by other authorities. When, as in the case in some large fever hospitals, the junior nurses are not engaged for any definite period, it is impossible to secure conscientious work. 
Their work too should be immediately supervised by sisters who have had a good general training of at least three years' duration.

The actual training consists, in addition to this supervision by the sisters of the ward, in lectures on anatomy and physiology, and later, on the infectious diseases themselves, each course being followed by an examination. Small practical classes in practical ward work, preparation for operations, bandaging, etc., are also held throughout the year.

It is, furthermore, necessary that the work of any isolation hospital shall be supervised by a resident Medical Officer. When a hospital, even of thirty or forty beds, is worked by a non-resident and often busy medical man, even though he be the Medical Officer of Health, and is nursed by frivolous or unintelligent women, the attainment of asepsis is very difficult, even though the building be of the most modern description. It is always possible to get a good staff if the salaries paid be adequate; but, as I said before, sanitary authorities do not believe in paying for a staft: their work is not evident, and they cannot be shown off. Even with the best of staff, however, the work will be done badly at times unless there is some motive common to all, namely, a feeling of patriotism for the hospital; and that, again, is hardly possible unless the period of adhesion to the hospital is a fairly long one. The nurses stay because they are being trained, but the rest of the staff can only be expected to remain if the salaries paid are adequate. Otherwise, directly they learn anything, they will obtain better-paid posts elsewhere. It is essential for this feeling of patriotism, not only to train your staff, but to keep them, and above all, by constant teaching to make all grades feel that the work of each person matters - has a direct effect on the patient. When they have learnt this they have next to feel that every one in an isolation hospital is playing a distinct part in the prevention of disease, and is not merely curing a succession of individual patients.

But you will say there is nothing new in these methods: this is only ordinary asepsis, which is as old as the hills. That is so; it is all quite obvious, and has been so -in general hospitals - for some time.

What $I$ do say, however, is that in isolation hospitals generally we are not sufficiently, I will even say efficiently, aseptic. Too often do sanitary authorities-leaving the vexed question of building out of the question for the moment, for I say frankly that I think they are of secondary, though yet of considerable importance-think that any kind of staff will do for a fever hospital, that infectious diseases can be treated, as they are tabulated, in bulk; in fact, that the hospital has served its purpose when it has removed the disease from the community. 
In very truth this is not so. They are bound not only to isolate disease, but to treat it. To secure asepsis in a general hospital is difficult enough, but in an atmosphere of virulent infection the difficulty is increased tenfold. The field of work as yet untouched is enormous. We are only on the threshold in the pathology of scarlet and enteric fevers. What record of our work, moreover, have we given to the medical profession for the advancement of learning? Time after time one sees good work, often very good work, done from morn till night and the world no better for it. We have barely time to observe, and none at all to record. We have only to turn to America to see what things might become. There the fever hospitals are on a footing with the general hospitals. Each has a crowd of willing workers, mostly unpaid by the bye, for they take the work as part of their training, and everything is not only investigated, but recorded. What we want is that young, recentlyqualified men shall come to us and work in numbers at the isolation hospitals, but we must first show that we have something to give them.

THe new Public Health Laboratory in connection with the University of Manchester was opened on January 27th. Congratulatory addresses were delivered by Earl Spencer (the Chancellor), Mr. W. J. Crossley, Sir William Church, and others. The laboratories are particularly good, and owe their inception to Professor Delépine, who is to be congratulated on the result of his labours.

To Enhance Resisting Power to Infection.-H. Miyake's experiments to increase resistance to infectious processes have been numerous. His final conclusion (Mitheilungen a. d. Grenzgebieten, Jena, xiii. 4 and 5) is to the effect that subcutaneous injection of nucleic acid has a remarkable effect in summoning the leucocytes to the spot, and reducing the danger from operative intervention. The dose for an adult is $50 \mathrm{cc}$. of a 2 per cent neutralized solution of yeast nucleinic acid. The $\cdot 5$ per cent solution is preferable, but as this requires $200 \mathrm{ce}$. of fluid, the more concentrated solution is generally used. No bad secondary effects were noticed in the 38 cases in which Mikulicz had used it at date of writing.

The Estrmation of Carbon Monoxide.-M. Albert Levy and M. Pécoul have devised a test which will show the presence of carbonmonoxide in the proportion of 1 part in 100,000 parts of air. The method is as follows: The suspected air is passed over per-iodic acid contained in a $\mathbf{U}$ tube kept at a temperature of $80^{\circ} \mathrm{O}$. The carbon-monoxide if present decomposes the per-iodic acid, setting free iodine; they then pass the air and the iodine through chloroform, which takes up the latter. The amount of iodine is estimated by a colorimeter scale, and from the amount of iodine the quantity of carbon-monoxide is deduced. The method is absolutely accurate, and no other element present in the atmosphere will rednce per-iodic acid. By its aid the atmosphere in the neighbourhood of a suspected stove can easily be examined in plenty of time for the necessary precautions to be taken. 